\title{
Effect of organic and bio-fertilization treatments on Fennel plant under drip irrigation system in Bahria Oases.
}

\section{II- Oil productivity and some chemical compounds}

\author{
Abdou, M.A.H.", Helmy, T.A.*, Salam M. S. , Abdel-Rahim, A.F.A. ${ }^{* *}$ and Hassan, A.A.* \\ "Horticultural department, Fac. of Agric., Minia Univ., Egypt. \\ **Agricultural Experiments \& Research Center, Minia Univ., Egypt.
}

Received on: 10/11/2020

Accepted on: 28/11/2020

\begin{abstract}
This experiment was carried out at the farm of Royal Herbs Company - Bahria Oases - Giza in a newly reclaimed desert land under the drip irrigation system during two successive seasons 2018/2019 and 2019/2020 with the aim of studying the effect of organic fertilizer (compost) and bio fertilization treatments \{phosphorein (PHOS) - Effective microorganisms (EM) - Minia azoteine (MA) and their combinations $\}$ and their interaction on oil production and some chemical compounds, namely, photosynthetic pigments (chlorophyll a, b and carotenoids) and nutrients percentage (N, $\mathrm{P}$ and $\mathrm{K} \%$ ) on fennel plants. The obtained results indicated that the application of compost significantly increased oil productivity in the seeds (oil\%, oil yield per plant and per feddan) and the studied chemical components (chlorophyll a, $\mathrm{b}$ and carotenoids; and $\mathrm{N}, \mathrm{P}$ and $\mathrm{K} \%$ ), the best treatment was 20-ton compost/fed.

All studied parameters were significantly affected by bio-fertilization; the best treatment was PHOS + EM + MAAslo, the interaction effect between the two factors was significant and . the best interaction treatment was compost at 15 or 20 ton/fed. with (PHOS + EM + MA) of bio-fertilization in the two seasons.
\end{abstract}

KEYWORDS: Compost, Bio-fertilization, Fennel, Oil productivity and Chemical composition.

\section{INTRODUCTION}

Even though herbs had been priced for their medicinal, flavoring and aromatic qualities for centuries, the imitation products of the modern age surpassed their value for a while. However, the unsighted dependence on synthetics was trouncing all the barricades and incite people to come again to the naturals with optimism of safety and sanctuary (Mahmoud et al., 2016).

Medicinal plants are used to cure many ailments that either non-curable or seldomly cured through modern systems of medicine. Approximately $80 \%$ of the world population depends on medicinal plants for their health and healing (Aliyu, 2003).

Fennel (Foeniculum vulgare, Miller) plant is one of the most common and widely cultivated aromatic and medicinal plants in middle Egypt Governorates such as BeniSuef, Minia and Assuit. It is a $120-180 \mathrm{~cm}$ long winter annual herb belonging to Fam. Apiaceae. Fennel is originally native to Mediterranean Sea region from where its cultivation spread out to Europe and Latin America Countries. The fruits which are the used part of fennel plant contain 3.5 - 5\% volatile oil with the most important components being anethole and fenchone. The fruits are commonly used in medicinal folklore and bakery and the volatile oil is involved in many pharmaceutical purposes and food industry (Badran et al., 2016).

The essential oil is used in cosmetics and pharmaceutical products (Lawrence, 1984; Braun and Franz, 1990).

Organic manures are important for medicinal and aromatic plants to produce the best product in both quantity and quality and it is also very safe for human health and environment. This is made by recycling organic material as plant and animals waste and food scraps in a controlled process. Continuous usage of inorganic fertilizer affects soil structure. Hence, organic manures can serve as alternative to mineral fertilizers for improving soil structure (Dauda et al., 2008) and microbial biomass (Suresh et al., 2004).

Bio-fertilizers are considered to be low cost, eco-friendly and renewable sources of plant nutrients supplementing chemical fertilizers in sustainable agricultural system. This refers to microorganism, which increase crop growth through different mechanisms, i.e. biological nitrogen fixation, phosphate-dissolving, growth promoting or hormonal substances, as well as, increasing availability of soil nutrients (Hedge et al., 1999).

Therefore, the aim of the present work was to study the effect of compost and bio-fertilization 
treatments, as well as, their interactions on essential oil productivity and chemical composition of fennel (Foeniculum vulgare, Mill.) plants.

\section{MATERIALS AND METHODS}

The present study was carried out for two seasons (2018/2019 and 2019/2020) at Bahria Oases (Gizza) - in Royal Herbs farm.

The fruits of fennel (Foeniculum vulgare, Mill.) plants were obtained from Royal Herbs company, Gizza, Shabramant. The experiment was arranged in a randomized complete block design in a split plot design with three replicates. The main plots (A) included four levels of compost, $(0,10,15$ and 20 ton/fed.) while seven treatments of bio-fertilization: Phosphorein (PHOS), Effective microorganisms (EM), Minia azotein (MA), $(\mathrm{PHOS}+\mathrm{EM}),(\mathrm{PHOS}+$ $\mathrm{MA}),(\mathrm{PHOS}+\mathrm{EM}+\mathrm{MA})$ and control treatments occupied the sub-plots (B).

Table 1. Physical and chemical properties of the used soil.

\begin{tabular}{llll}
\hline Soil character & Values & Soil character & Values \\
\hline Chemical properties: & & Available nutrients: & 116.69 \\
\hline $\mathrm{pH} 1: 2.5$ & 7.80 & $\mathrm{Ca}^{++}(\mathrm{ppm})$ & 3.77 \\
E.C. $(\mathrm{dS} / \mathrm{m})$ & 0.77 & $\mathrm{Mg}^{++}(\mathrm{ppm})$ & 35.50 \\
$\mathrm{O} . \mathrm{M}$. & 0.13 & $\mathrm{Na}^{+}(\mathrm{ppm})$ & 15.56 \\
$\mathrm{CaCO}$ & 3.35 & $\mathrm{~K}^{+}(\mathrm{ppm})$ & 93.70 \\
Exchangeable nutrients: & & Physical properties: & 3.85 \\
$\mathrm{Ca}^{++}(\mathbf{m g} / \mathbf{1 0 0}$ g soil) & 3.5 & Sand $(\%)$ & 2.45 \\
$\mathrm{Mg}^{++}(\mathbf{m g} / \mathbf{1 0 0}$ g soil) & 2.5 & Silt $(\%)$ & Sandy \\
$\mathrm{Na}^{+}(\mathbf{m g} / \mathbf{1 0 0}$ g soil) & 0.8 & Clay $(\%)$ & \\
$\mathrm{K}^{+}(\mathbf{m g} / \mathbf{1 0 0}$ g soil) & 0.2 & Soil type & \\
\hline
\end{tabular}

Fresh and active bio-fertilizers, Minia azotein and effective microorganisms E.M. (containing Nfixing bacteria) and Phosphorein (containing phosphate dissolving bacteria) were obtained from the Laboratory of Bio-fertilizers, Department of Genetic, Fac. of Agric., Minia University. Biofertilizers were applied three times to the soil beside the plants at the rate of $50 \mathrm{~cm}^{3} /$ hill $\left(1 \mathrm{ml}=10^{7}\right.$ cells of Table (2): Physical and chemical properties of the used compost:

\begin{tabular}{lll}
\hline Properties & First season & Second season \\
\hline Organic matter $(\%)$ & 15.00 & 13.80 \\
Humidity $(\%)$ & 7.90 & 9.00 \\
Ca $(\mathbf{p p m})$ & 1405.10 & 1295.00 \\
Mg $(\mathbf{p p m})$ & 46.60 & 47.40 \\
Na $(\mathbf{p p m})$ & 644.00 & 613.00 \\
K $(\mathbf{p p m})$ & 476.20 & 485.10 \\
$\mathbf{P}(\mathbf{p p m})$ & 4.30 & 4.70 \\
E.C. $(\mathbf{d S} / \mathbf{m})$ & 6.21 & 6.65 \\
pH & 8.10 & 7.97 \\
\hline
\end{tabular}

The sub plot treatments (B) were as follows: $\mathbf{b}_{1}$, Control; $\mathbf{b}_{2}$, Phosphorien; $\mathbf{b}_{3}$, Effective microorganism; $\mathbf{b}_{\mathbf{4}}$, Minia azotein; $\mathbf{b}_{5}$, biofertilizers (Phosphorien+ Effective microorganism); b6, biofertilizers (Phosphorien + Minia Azotein); $\mathbf{b}_{7}$,
Therefore, the interaction treatments $\left(A^{*} B\right)$ were 28 treatments. Fennel fruits were sown in October $21^{\text {st }}$ in the two growth seasons in plots. Each plot consists of $4 \mathrm{~m}$ width $\mathrm{x} 7 \mathrm{~m}$ length and it contains 7 terraces (2 line/terrace) with $1 \mathrm{~m}$ separation to prevent water seepage from each plot to adjacent plot. Planting rate was $5 \mathrm{~kg}$ seeds/fed. in hills with $50 \mathrm{~cm}$ apart between hills. Therefore, each experimental unit contained 224 plants (which were thinned into two plants per hill). Thus, the number of plants/fed. was 32,000 plants. Two weeks before planting date, compost was added during preparation of the soil for planting in the two experimental seasons at the Royal Herbs Farm. The physical and chemical analysis of the used soil in both seasons were determined according to Page et al. (1982) and shown in Table (1).

bacteria). The first dose; for Phosphorein, Effective microorganism and Minia azotein was added 40 days from sowing date, 20 days interval between the three doses and then plants were irrigated immediately. The Compost was obtained from El-Sharqia company. The physical and chemical properties of the used compost are shown in Table (2).

biofertilizers (Phosphorien + Effective microorganism + Minia azotein).

The following data were recorded at the harvesting time except chlorophyll a, b and 
carotenoids were recorded after 20 days from the last experimental treatments (bio-fertilization).

\subsection{The essential oil productivity:}

Essential oil percentage (\%), essential oil yield/plant (ml) and essential oil yield/fed. (liter).

Essential oil percentage was determined in dried samples in both seasons by subjecting to hydro distillation in Clevenger apparatus according to method described by the Egyptian Pharmacopoeia (1984), the resulted oil was dried over anhydroussoduim sulphate and kept at refrigerator until Gas Liquid Chromatography Analyses. Then the essential oil yield per plant and per feddan was calculated.

\subsection{Chemical composition:}

2.2.1. Chlorophyll a, b and carotenoids (mg/g fresh wight of herbs).

Chlorophyll a, b and carotenoids were determined in fresh herbs samples (mg/g f.w.) according to the method cited from Fadl and Sari ElDeen (1978).

\subsubsection{Elements percentages ( $\mathrm{N}, \mathrm{P}$ and $\mathrm{K})$.}

To determine $\mathrm{N}, \mathrm{P}$ and $\mathrm{K} \%$ in plant tissues, $0.2 \mathrm{~g}$ crude dried herbs powder from each sample was wet digested with a mixture of concentrated sulphoric
$\left(\mathrm{H}_{2} \mathrm{SO}_{4}\right)$ and perchloric $\left(\mathrm{HClPO}_{4}\right)$ acids, then heated until become clear solution. This solution was quantitavely transferred into $100 \mathrm{ml}$ measuring flask and kept for determinations. (Cottenie, 1982).

\subsection{Statistical analysis:}

All obtained data in the first and second seasons were tabulated and statistically analyzed according to MSTAT-C (1986) and the L.S.D. test at $5 \%$ was followed to compare between the means.

\section{RESULTS AND DISCUSSIONS}

\subsection{The essential oil productivity}

It is clearly noticed from data presented in Tables ( 3 and 4 ) that plants of fennel which received compost at 20 ton/fed. showed the highest significant increase of essential oil (\%), oil yield/plant (ml/plant) and oil yield/fed. (liter) followed by compost at 15 ton/fed. then 10 ton/fed. in both seasons.

These results are in accordance with those obtained by Badran and Safwat (2004), Abdou et al. (2009b), Azzaz et al. (2009), Moradi et al. (2011), Abdou et al. (2012), Jamshidi et al. (2012), Younesian et al. (2013), Abarghouei (2014), Eisa (2016) and Abd El-Aleem et al. (2017) on fennel plants.

Table 3. Effect of compost and bio-fertilization, as well as, their combination treatments on oil (\%) and oil yield/plant (ml) of Foeniculum vulgare, Mill. plants during the first and second seasons.

\begin{tabular}{|c|c|c|c|c|c|c|c|c|c|c|}
\hline \multirow{3}{*}{$\begin{array}{l}\text { Bio-fertilization } \\
\text { treatments (B) }\end{array}$} & \multicolumn{10}{|c|}{ Compost levels ton/fed. (A) } \\
\hline & $\mathbf{0}$ & 10 & 15 & 20 & $\begin{array}{c}\text { Mean } \\
\text { (B) }\end{array}$ & $\mathbf{0}$ & 10 & 15 & 20 & $\begin{array}{c}\text { Mean } \\
\text { (B) }\end{array}$ \\
\hline & \multicolumn{5}{|c|}{ The $1^{\text {st }}$ season $(2018 / 2019)$} & \multicolumn{5}{|c|}{ The $2^{\text {nd }}$ season $(2019 / 2020)$} \\
\hline \multicolumn{11}{|c|}{ Oil (\%) } \\
\hline Control & 1.45 & 2.01 & 2.24 & 2.45 & 2.04 & 1.52 & 2.10 & 2.34 & 2.57 & 2.13 \\
\hline PHOS & 1.53 & 2.07 & 2.53 & 2.62 & 2.19 & 1.60 & 2.17 & 2.65 & 2.74 & 2.29 \\
\hline EM & 1.69 & 2.30 & 2.85 & 2.90 & 2.44 & 1.77 & 2.41 & 2.98 & 3.03 & 2.55 \\
\hline MA & 1.60 & 2.17 & 2.70 & 2.77 & 2.31 & 1.67 & 2.27 & 2.82 & 2.89 & 2.41 \\
\hline PHOS+EM & 1.86 & 3.08 & 3.33 & 3.43 & 2.92 & 1.94 & 3.22 & 3.49 & 3.59 & 3.06 \\
\hline PHOS+MA & 1.76 & 2.39 & 3.00 & 3.14 & 2.57 & 1.84 & 2.49 & 3.13 & 3.29 & 2.69 \\
\hline PHOS+EM+MA & 1.92 & 3.23 & 3.51 & 3.61 & 3.07 & 2.01 & 3.37 & 3.67 & 3.77 & 3.21 \\
\hline Mean (A) & 1.69 & 2.46 & 2.88 & 2.99 & & 1.77 & 2.58 & 3.01 & 3.12 & \\
\hline L.S.D. at $5 \%$ & A: 0.05 & & B: 0.09 & & 0.18 & A: 0 & & B: 0.12 & & 3: 0.24 \\
\hline \multicolumn{11}{|c|}{ Oil yield/plant (ml/plant) } \\
\hline Control & 0.32 & 0.49 & 0.60 & 0.82 & 0.56 & 0.35 & 0.54 & 0.65 & 0.90 & 0.61 \\
\hline PHOS & 0.34 & 0.53 & 0.86 & 0.95 & 0.67 & 0.37 & 0.58 & 0.94 & 1.05 & 0.74 \\
\hline EM & 0.39 & 0.67 & 1.13 & 1.18 & 0.84 & 0.43 & 0.74 & 1.24 & 1.29 & 0.93 \\
\hline MA & 0.36 & 0.57 & 0.99 & 1.07 & 0.75 & 0.39 & 0.63 & 1.09 & 1.18 & 0.82 \\
\hline PHOS+EM & 0.44 & 1.33 & 1.68 & 1.76 & 1.30 & 0.48 & 1.46 & 1.85 & 1.93 & 1.43 \\
\hline PHOS+MA & 0.41 & 0.70 & 1.27 & 1.49 & 0.97 & 0.45 & 0.77 & 1.39 & 1.64 & 1.06 \\
\hline PHOS+EM+MA & 0.46 & 1.58 & 1.86 & 1.93 & 1.46 & 0.51 & 1.73 & 2.04 & 2.12 & 1.60 \\
\hline Mean (A) & 0.39 & 0.84 & 1.20 & 1.31 & & 0.43 & 0.92 & 1.32 & 1.44 & \\
\hline L.S.D. at $5 \%$ & A:0.08 & & B:0.09 & & B:0.18 & A:0. & & B:0.10 & & B:0.20 \\
\hline
\end{tabular}


Table 4. Effect of compost and bio-fertilization, as well as, their combination treatments on oil yield/fed. (liter) of Foeniculum vulgare, Mill. plants during the first and second seasons.

\begin{tabular}{|c|c|c|c|c|c|c|c|c|c|c|}
\hline \multirow{3}{*}{$\begin{array}{l}\text { Bio-fertilization } \\
\text { treatments (B) }\end{array}$} & \multicolumn{10}{|c|}{ Compost levels ton/fed. (A) } \\
\hline & $\mathbf{0}$ & 10 & 15 & 20 & $\begin{array}{c}\text { Mean } \\
\text { (B) }\end{array}$ & $\mathbf{0}$ & 10 & 15 & 20 & $\begin{array}{c}\text { Mean } \\
\text { (B) }\end{array}$ \\
\hline & \multicolumn{5}{|c|}{ The $1^{\text {st }}$ season $(2018 / 2019)$} & \multicolumn{5}{|c|}{ The $2^{\text {nd }}$ season $(2019 / 2020)$} \\
\hline \multicolumn{11}{|c|}{ Oil yield/fed. (liter) } \\
\hline Control & 10.24 & 15.68 & 19.20 & 26.24 & 17.92 & 11.20 & 17.28 & 20.80 & 28.80 & 19.52 \\
\hline PHOS & 10.88 & 16.96 & 27.52 & 30.40 & 21.44 & 11.84 & 18.56 & 30.08 & 33.60 & 23.68 \\
\hline EM & 12.48 & 21.44 & 36.16 & 37.76 & 26.88 & 13.76 & 23.68 & 39.68 & 41.28 & 29.76 \\
\hline MA & 11.52 & 18.24 & 31.68 & 34.24 & 24.00 & 12.48 & 20.16 & 34.88 & 37.76 & 26.24 \\
\hline PHOS+EM & 14.08 & 42.56 & 53.76 & 56.32 & 41.60 & 15.36 & 46.72 & 59.20 & 61.76 & 45.76 \\
\hline PHOS+MA & 13.12 & 22.40 & 40.64 & 47.68 & 31.04 & 14.40 & 24.64 & 44.48 & 52.48 & 33.92 \\
\hline PHOS+EM+MA & 14.72 & 50.56 & 59.52 & 61.76 & 46.72 & 16.32 & 55.36 & 65.28 & 67.84 & 51.20 \\
\hline Mean (A) & 12.48 & 26.88 & 38.40 & 41.92 & & 13.76 & 29.44 & 42.24 & 46.08 & \\
\hline L.S.D. at $5 \%$ & A: $\mathbf{2 . 8 0}$ & & B: 2.30 & & $: 4.60$ & A: $\mathbf{2 . 8 0}$ & & B: 2.38 & & 4.67 \\
\hline
\end{tabular}

PHOS: Phosphorein

EM: Effective microorganisms

MA: Minia azotein

The data presented in tables (3 and 4) indicated that essential oil (\%), oil yield/plant (ml/plant) and oil yield/fed. (liter) in the fruits of fennel plants was improved as a result of inoculating the growing soil with any commercial product of biofertilizers either separately or collectively used. Inoculating soil with Phosphorein plus Effective microorganisms plus Minia azotein gave significantly higher oil (\%) and oil yield (ml/plant and liter/fed.) compared with all used treatments. The lowest essential oil percentage was obtained by the control treatment in both seasons.

Similar results were obtained by Sharaf and Khattab (2004), Abdou et al. (2009b) and Gamar et al. (2018) on fennel plants, Abd El-Latif (2002), AlShareif (2006) and Abdou et al. (2009a) on caraway plants, Badran et al. (2003), Hemdan (2008) and Zand et al. (2013) on anise plants, Hellal et al. (2011) on dill, Rekaby (2013) and Mounika et al. (2018) on coriander plants.

The interaction between compost and biofertilization treatments was significant for oil (\%), oil yield/plant (ml/plant) and oil yield/fed (liter/fed.) of fennel in both seasons as clearly shown in tables (3 and 4).

The best interaction treatments were obtained by adding compost at 20 ton/fed. in combination with (PHOS + EM + MA) or adding compost at 20 ton/fed. plus $(\mathrm{PHOS}+\mathrm{EM})$.

\subsection{Chemical composition:}

Data presents in Tables $(5,6$ and 7$)$ indicated that, the content of chlorophyll a, b and carotenoids in the fresh weight of herbs and N, P and K\% in dry weight of herbs of fennel plants were greatly affected by compost treatments as compared to control treatment in both seasons. The high contents were obtained with 20 ton/fed. compost.

Similar results were obtained by Tanious (2008) and Abdou et al. (2012) on fennel plants, Ahmed (2017), Hassan (2008) and Mahmoud (2009) on black cumin.

According to data tabulated in Tables $(5,6$ and 7 ), it could be concluded that the bio-fertilizers compound progressively increased contents of chlorophyll a, b, carotenoids, N, P and $\mathrm{K} \%$ as compared with control treatment in both seasons. So, the highest contents of the three photosynthetic pigments and $\mathrm{N}, \mathrm{P}$ and $\mathrm{K}$ were obtained with treatments (PHOS + EM + MA).

The above results of contents of pigments are in paralled with those obtained by Muthaura et al. (2010) on pigweed plants, Patil (2010) on Stevia rebaudiana var. Bertoni, Ibrahim (2014) on Khilla and Ahmed (2017) on black cumin plants.

The interaction between compost and biofertilization treatments was significant for chlorophyll a, b, carotenoids, $\mathrm{N}, \mathrm{P}$ and $\mathrm{K} \%$ in both seasons as shown in Tables (5, 6 and 7).

The best interaction effects between compost and bio-fertilization treatments were obtained by supplying fennel plants with compost at 20 ton/fed. and inoculation with (PHOS + EM + MA) or plus (PHOS + EM in some cases), also by adding compost at 15 ton/fed. + (PHOS + EM + MA $)$. 
Scientific Journal of Agricultural Sciences 2 (2): 72-79, 2020

Table 5. Effect of compost and bio-fertilization, as well as, their combination treatments on chlorophyll a and b (mg/g F.W.) of Foeniculum vulgare, Mill. plants during the first and second seasons.

\begin{tabular}{|c|c|c|c|c|c|c|c|c|c|c|}
\hline \multirow{3}{*}{$\begin{array}{l}\text { Bio-fertilization } \\
\text { treatments }(B)\end{array}$} & \multicolumn{10}{|c|}{ Compost levels, ton/fed. (A) } \\
\hline & $\mathbf{0}$ & 10 & 15 & 20 & $\begin{array}{c}\text { Mean } \\
\text { (B) }\end{array}$ & $\mathbf{0}$ & 10 & 15 & 20 & $\begin{array}{c}\text { Mean } \\
\text { (B) }\end{array}$ \\
\hline & \multicolumn{5}{|c|}{ The $1^{\text {st }}$ season $(2018 / 2019)$} & \multicolumn{5}{|c|}{ The $2^{\text {nd }}$ season $(2019 / 2020)$} \\
\hline \multicolumn{11}{|c|}{ Chlorophyll a (mg/ g F.W.) } \\
\hline Control & 1.569 & 1.810 & 1.897 & 1.978 & 1.813 & 1.612 & 1.862 & 1.952 & 2.036 & 1.865 \\
\hline PHOS & 1.612 & 1.813 & 1.981 & 2.016 & 1.855 & 1.662 & 1.865 & 2.039 & 2.074 & 1.910 \\
\hline EM & 1.624 & 1.905 & 2.265 & 2.268 & 2.016 & 1.670 & 1.960 & 2.329 & 2.332 & 2.073 \\
\hline MA & 1.621 & 1.833 & 2.236 & 2.250 & 1.985 & 1.668 & 1.885 & 2.300 & 2.317 & 2.042 \\
\hline PHOS+EM & 1.778 & 2.340 & 2.448 & 2.477 & 2.261 & 1.827 & 2.407 & 2.517 & 2.549 & 2.325 \\
\hline PHOS+MA & 1.673 & 1.943 & 2.271 & 2.342 & 2.057 & 1.720 & 1.998 & 2.337 & 2.410 & 2.116 \\
\hline PHOS+EM+MA & 1.807 & 2.393 & 2.480 & 2.509 & 2.297 & 1.859 & 2.459 & 2.552 & 2.581 & 2.363 \\
\hline Mean (A) & 1.669 & 2.005 & 2.225 & 2.263 & & 1.717 & 2.062 & 2.289 & 2.328 & \\
\hline L.S.D. at $5 \%$ & \multicolumn{2}{|c|}{ A: $\mathbf{0 . 0 3 3}$} & B: 0.025 & \multicolumn{2}{|c|}{ AB: $\mathbf{0 . 0 5 0}$} & \multicolumn{2}{|c|}{ A: $\mathbf{0 . 0 3 7}$} & B: 0.028 & \multicolumn{2}{|c|}{ AB: 0.056} \\
\hline \multicolumn{11}{|c|}{ Chlorophyll b (mg/ g F.W.) } \\
\hline Control & 0.541 & 0.624 & 0.654 & 0.682 & 0.625 & 0.556 & 0.642 & 0.673 & 0.702 & 0.643 \\
\hline PHOS & 0.556 & 0.625 & 0.683 & 0.695 & 0.640 & 0.573 & 0.643 & 0.703 & 0.715 & 0.658 \\
\hline EM & 0.560 & 0.657 & 0.781 & 0.781 & 0.695 & 0.576 & 0.676 & 0.803 & 0.804 & 0.715 \\
\hline MA & 0.559 & 0.632 & 0.771 & 0.776 & 0.685 & 0.575 & 0.650 & 0.793 & 0.799 & 0.705 \\
\hline PHOS+EM & 0.613 & 0.807 & 0.844 & 0.854 & 0.779 & 0.630 & 0.830 & 0.868 & 0.879 & 0.802 \\
\hline PHOS+MA & 0.577 & 0.670 & 0.783 & 0.807 & 0.709 & 0.593 & 0.689 & 0.806 & 0.831 & 0.730 \\
\hline PHOS+EM+MA & 0.623 & 0.825 & 0.855 & 0.865 & 0.792 & 0.641 & 0.848 & 0.880 & 0.890 & 0.815 \\
\hline Control & 0.576 & 0.691 & 0.767 & 0.780 & & 0.592 & 0.711 & 0.790 & 0.803 & \\
\hline L.S.D. at $5 \%$ & \multicolumn{2}{|c|}{ A: 0.009} & B: 0.008 & \multicolumn{2}{|c|}{ AB: 0.016} & \multicolumn{2}{|c|}{ A: 0.013} & B: 0.010 & \multicolumn{2}{|c|}{ AB: 0.020} \\
\hline
\end{tabular}

PHOS: Phosphorein

EM: Effective microorganisms

MA: Minia azotein

Table 6. Effect of compost and bio-fertilization, as well as, their combination treatments on carotenoids (mg/g F.W.) and nitrogen percentage of Foeniculum vulgare, Mill. plants during the first and second seasons.

\begin{tabular}{|c|c|c|c|c|c|c|c|c|c|c|}
\hline \multirow{3}{*}{$\begin{array}{l}\text { Bio-fertilization } \\
\text { treatments }(B)\end{array}$} & \multicolumn{10}{|c|}{ Compost levels ton/fed. (A) } \\
\hline & $\mathbf{0}$ & 10 & 15 & 20 & $\begin{array}{c}\text { Mean } \\
\text { (B) }\end{array}$ & $\mathbf{0}$ & 10 & 15 & 20 & $\begin{array}{c}\text { Mean } \\
\text { (B) }\end{array}$ \\
\hline & \multicolumn{5}{|c|}{ The $1^{\text {st }}$ season $(2018 / 2019)$} & \multicolumn{5}{|c|}{ The $2^{\text {nd }}$ season $(2019 / 2020)$} \\
\hline \multicolumn{11}{|c|}{$\begin{array}{l}\text { Carotenoids (mg/g F.W.) } \\
\end{array}$} \\
\hline Control & 0.661 & 0.763 & 0.799 & 0.834 & 0.764 & 0.680 & 0.785 & 0.823 & 0.858 & 0.786 \\
\hline PHOS & 0.680 & 0.764 & 0.835 & 0.849 & 0.782 & 0.700 & 0.786 & 0.859 & 0.874 & 0.805 \\
\hline EM & 0.684 & 0.803 & 0.955 & 0.957 & 0.850 & 0.704 & 0.826 & 0.981 & 0.983 & 0.874 \\
\hline MA & 0.683 & 0.772 & 0.942 & 0.948 & 0.837 & 0.703 & 0.794 & 0.969 & 0.977 & 0.861 \\
\hline PHOS+EM & 0.749 & 0.986 & 1.032 & 1.044 & 0.953 & 0.770 & 1.014 & 1.061 & 1.074 & 0.980 \\
\hline PHOS+MA & 0.705 & 0.819 & 0.983 & 0.990 & 0.874 & 0.725 & 0.842 & 0.985 & 1.016 & 0.892 \\
\hline PHOS+EM+MA & 0.761 & 1.008 & 1.045 & 1.057 & 0.968 & 0.783 & 1.036 & 1.076 & 1.088 & 0.996 \\
\hline Mean (A) & 0.703 & 0.845 & 0.942 & 0.954 & & 0.724 & 0.869 & 0.965 & 0.981 & \\
\hline L.S.D. at $5 \%$ & \multicolumn{2}{|c|}{ A: 0.008} & B: 0.011 & \multicolumn{2}{|c|}{ AB: 0.022} & \multicolumn{2}{|c|}{ A: 0.010} & B: 0.012 & \multicolumn{2}{|c|}{ AB: 0.024} \\
\hline \multicolumn{11}{|c|}{ Nitrogen $(\%)$} \\
\hline Control & 2.21 & 2.70 & 2.86 & 3.05 & 2.71 & 2.40 & 2.88 & 3.01 & 3.24 & 2.88 \\
\hline PHOS & 2.27 & 2.75 & 3.12 & 3.19 & 2.83 & 2.49 & 2.94 & 3.30 & 3.36 & 3.02 \\
\hline EM & 2.42 & 2.93 & 3.37 & 3.44 & 3.04 & 2.63 & 3.11 & 3.54 & 3.60 & 3.22 \\
\hline MA & 2.35 & 2.81 & 3.25 & 3.31 & 2.93 & 2.56 & 2.99 & 3.42 & 3.47 & 3.11 \\
\hline PHOS+EM & 2.56 & 3.58 & 3.78 & 3.86 & 3.45 & 2.74 & 3.71 & 3.90 & 3.98 & 3.58 \\
\hline PHOS+MA & 2.50 & 2.98 & 3.51 & 3.65 & 3.16 & 2.68 & 3.22 & 3.64 & 3.77 & 3.33 \\
\hline PHOS+EM+MA & 2.64 & 3.72 & 3.91 & 3.98 & 3.56 & 2.80 & 3.83 & 4.02 & 4.08 & 3.68 \\
\hline Mean (A) & 2.42 & 3.07 & 3.40 & 3.50 & & 2.61 & 3.24 & 3.55 & 3.64 & \\
\hline L.S.D. at $5 \%$ & A: 0. & & B: 0.05 & & 0.10 & A: 0. & & B: 0.07 & & 0.14 \\
\hline
\end{tabular}

PHOS: Phosphorein

EM: Effective microorganisms

MA: Minia azotein 
Table 7. Effect of compost and bio-fertilization, as well as, their combination treatments on phosphorus and potassium percentages of Foeniculum vulgare, Mill. plants during the first and second seasons.

\begin{tabular}{|c|c|c|c|c|c|c|c|c|c|c|}
\hline \multirow{3}{*}{$\begin{array}{l}\text { Bio-fertilization } \\
\text { treatments }(B)\end{array}$} & \multicolumn{10}{|c|}{ Compost levels ton/fed. (A) } \\
\hline & $\mathbf{0}$ & 10 & 15 & 20 & $\begin{array}{c}\text { Mean } \\
\text { (B) }\end{array}$ & $\mathbf{0}$ & 10 & 15 & 20 & $\begin{array}{c}\text { Mean } \\
\text { (B) }\end{array}$ \\
\hline & \multicolumn{5}{|c|}{ The $1^{\text {st }}$ season $(2018 / 2019)$} & \multicolumn{5}{|c|}{ The $2^{\text {nd }}$ season $(2019 / 2020)$} \\
\hline \multicolumn{11}{|c|}{ Phosphorus (\%) } \\
\hline Control & 0.216 & 0.276 & 0.301 & 0.327 & 0.280 & 0.241 & 0.307 & 0.335 & 0.361 & 0.311 \\
\hline PHOS & 0.225 & 0.284 & 0.338 & 0.348 & 0.299 & 0.257 & 0.315 & 0.364 & 0.377 & 0.328 \\
\hline EM & 0.242 & 0.311 & 0.375 & 0.384 & 0.328 & 0.277 & 0.343 & 0.405 & 0.412 & 0.359 \\
\hline MA & 0.234 & 0.292 & 0.357 & 0.366 & 0.312 & 0.268 & 0.324 & 0.388 & 0.395 & 0.344 \\
\hline PHOS+EM & 0.259 & 0.399 & 0.428 & 0.439 & 0.381 & 0.293 & 0.429 & 0.450 & 0.455 & 0.407 \\
\hline PHOS+MA & 0.250 & 0.320 & 0.393 & 0.410 & 0.343 & 0.284 & 0.352 & 0.423 & 0.439 & 0.375 \\
\hline PHOS+EM+MA & 0.268 & 0.419 & 0.448 & 0.460 & 0.399 & 0.301 & 0.444 & 0.466 & 0.475 & 0.422 \\
\hline Mean (A) & 0.242 & 0.329 & 0.377 & 0.391 & & 0.274 & 0.359 & 0.404 & 0.416 & \\
\hline L.S.D. at $5 \%$ & \multicolumn{2}{|c|}{ A: 0.009} & B: 0.006 & \multicolumn{2}{|c|}{ AB: 0.012} & \multicolumn{2}{|c|}{ A: 0.011} & B: 0.008 & \multicolumn{2}{|c|}{ AB: 0.016} \\
\hline \multicolumn{11}{|c|}{ Potassium (\%) } \\
\hline Control & 1.98 & 2.32 & 2.50 & 2.61 & 2.35 & 2.08 & 2.41 & 2.55 & 2.73 & 2.44 \\
\hline PHOS & 2.03 & 2.38 & 2.66 & 2.70 & 2.44 & 2.12 & 2.46 & 2.75 & 2.79 & 2.53 \\
\hline EM & 2.14 & 2.53 & 2.84 & 2.88 & 2.60 & 2.20 & 2.60 & 2.95 & 2.99 & 2.69 \\
\hline MA & 2.10 & 2.44 & 2.75 & 2.81 & 2.53 & 2.17 & 2.50 & 2.86 & 2.89 & 2.61 \\
\hline PHOS+EM & 2.24 & 2.97 & 3.12 & 3.20 & 2.88 & 2.31 & 3.06 & 3.21 & 3.26 & 2.96 \\
\hline PHOS+MA & 2.19 & 2.56 & 2.92 & 3.03 & 2.68 & 2.26 & 2.68 & 3.02 & 3.12 & 2.77 \\
\hline PHOS+EM+MA & 2.27 & 3.06 & 3.25 & 3.28 & 2.97 & 2.35 & 3.16 & 3.36 & 3.41 & 3.07 \\
\hline Mean (A) & 2.14 & 2.61 & 2.86 & 2.93 & & 2.21 & 2.70 & 2.96 & 3.03 & \\
\hline L.S.D. at $5 \%$ & \multicolumn{2}{|c|}{ A: 0.04} & B: 0.07 & \multicolumn{2}{|c|}{ AB: 0.14} & A: 0. & & B: 0.08 & \multicolumn{2}{|c|}{ AB: 0.16} \\
\hline $\begin{array}{l}\text { PHOS: Phosphore } \\
\text { EM: Effective mic } \\
\text { MA: Minia azotein }\end{array}$ & anisms & & & & & & & & & \\
\hline
\end{tabular}

\section{CONCLUSION}

It can be concluded that to obtain the best oil production and chemical compounds, namely, photosynthetic pigments (chlorophyll a, b and carotenoids) and nutrients percentage ( $\mathrm{N}, \mathrm{P}$ and $\mathrm{K} \%)$ of fennel, must be supplying plants combined with compost at 20 or 15 ton/fed. with Phosphoren + Effective microorganisms + Minia Azotein.

\section{REFERENCES}

Abarghouei HB (2014). An evaluation of the effect of plow and fertilizer types on qualitative and quantitative yields of fennel (Foeniculum vulgare, Mill.). Journal of Applied Science and Agriculture, 9(4): 1488-1493.

Abd El-Aleem W, Hendawy SF, Hamed ES, Toaima WIM (2017). Effect of planting dates, organic fertilization and foliar spray of algae extract on productivity of Dutch fennel plants under Sinai conditions. Journal of Medicinal Plants Studies. 5(3): 327-334.

Abd El-Latif TA (2002). Effect of organic manure and bio-fertilizer on caraway plants (Carum carvi, L.). J. Agric. Sci., Mansoura Univ., 27 (5): 3459 3468.
Abdou MAH, Attia FA, Ahmed ET, Abd ElNaeem L (2009a). Response of caraway plants to some organic, antioxidants and bio-fertilization treatments. Proc. $4^{\text {th }}$ Inter. Environ. Conf., Mansoura Univ. on Environmental and Healthy Safety, p: 109119.

Abdou MAH, Attia FA, Taha RA, Tanious C (2009b). Effect of some organic, bio-fertilization and antioxidant treatments on fennel plants. Proc. $5^{\text {th }}$ Inter. of Sustain, Agric. and Develop. Fac. of Agric., Fayoum Univ., 21-23. December, p: 159-178.

Abdou MAH, Taha RA, Abd El-Raaof RM, Salah El-Deen RM (2012). Response of fennel plants to organic, bio and mineral fertilization. Proc. Second Inter. Conf. Physiological, Microbiological and Ecological Plant Sciences, (April 29 ${ }^{\text {th }}-30^{\text {th }}$ ) Fac. of science, Minia Univ.

Ahmed EFA (2017). Evaluation of certain fertilizing programs on anise and black cumin plants. Ph.D. Thesis. Fac. Assiut Univ., Egypt.

Aliyu L (2003). Effect of manure type and rate on growth, yield and yield components of pepper. Journal of Sustainable Agriculture and the Environment. Vol. 5, p.: 92-98.

Al-Shareif AMO (2006). Response of caraway plants grown in sandy soil under drip irrigation 
system to some bio-fertilization and antioxidant treatments. M. Sc. Thesis. Fac. of Agric. Minia Univ. Egypt.

Azzaz NA, Hassan EA, Hamad EH (2009). The chemical constituent and vegetative and yielding characteristics of fennel plants treated with organic and bio-fertilizer instead of mineral fertilizer. Australian Journal of Basic and Applied Sciences, 3 (2): $579-587$.

Badran FS, Safwat MS (2004). Response of fennel plants to organic manure and bio-fertilizers in replacement of chemical fertilization. Egypt J. Agric. Res., 82 (2).

Badran FS Ahmad ET, El-Ghadban EA, Ayyat AM (2016). Effect of compost /NPK and biofertilization treatments on vegetative growth, yield and herb NPK\% of fennel plants. Scientific J. Flowers \& Ornamental Plants, 4 (2), p.: 175-185.

Badran FS, Attia FA, Ahmed ET, Soliman HA (2003). Effect of chemical and biological fertilization on growth, yield and oil production of anise (Pimpinella anisum, L.) plants. II-Effect of NP mineral/biofertilization and micronutrient treatments. Proc. Egyptian-Syrian 1st Conf., Minia Univ., Dec.

Braun M, Franz G (1990). Quality criteria of bitter fennel oil in the German pharmacopoeia. Pharm Pharmcoel letter: 9 (2): 48-51.

Cottenie A, Verloo M, Velghe M, Camerlynck $R$ (1982). Chemical Analysis of Plant and Soil. Laboratory of Analytical and Ayro Chemistry. State Univ. Ghent, Belgium.

Dauda SN, Ajayi FA, Ndor E (2008). Growth and yield of watermelon (Citrullus lanatus) as affected by Poultry manure application. J. Agric. Soc. Sci., 4: 121 $-211$.

Egyptian Pharmacopoeia (1984). Egyptian Pharmacopoeia, General Organization for Governmental. Printing Office, Ministry of Health, Cairo, Egypt, p.: 31-33.

Eisa EA (2016). Effect of different sources of organic fertilizers and seaweed extract on growth and essential oil of sweet fennel (Foeniculum vulgare, mill.) plants. J. Plant Production, Mansoura Univ. 7(6): $575-584$.

Fadl MS, Sari El-Deen SA (1978) Effects of Nbenzyl adenine on photosynthetic pigments and total soluble sugars on olive seedlings growth under saline condition. Research Bulletin No. 843, Fac. of Agric. Sci Ain Shams Univ. 29 (1): 19-28.

Gamar PB1, Mevada KD, Ombase KC, Dodiya CJ (2018). Response of drilled rabi fennel (Foeniculum vulgare mill.) to integrated nutrient management practices. International Journal of Agriculture Sciences. 10(2), p: 4995-4998.

Hassan RF (2008). Growth and yield of three black cumin (Nigella sativa, L.) londraces as affected by organic and inorganic fertilization. M.Sc. Thesis, Faculty of Agriculture, Assiut University, Egypt.

Hedge DM, Dwivedi BS, Sudhakara BSS (1999). Biofertilizers for cereal production in India - A review. Indian J. Agric. Res., 69 (2): 73-83.

Hellal FA, Mahfouz SA, Hassan FAS (2011). Partial substitution of mineral nitrogen fertilizer by bio-fertilizer on Anethum graveolens, L. plant Agric. Biol. J. N. Am., 2 (4): 652-660.

Hemdan SHO (2008). Effect of some organic and bio-fertilization treatments on anise plants. M. Sc. Thesis, Fac. Agric. Minia Univ., Egypt.

Ibrahim TIEI (2014). Influence of some agricultural treatments on Ammi visnaga plants. Ph. D. Thesis, Fac. of Agric. Minia Univ.

Jamshidi E, Ghalavand A, Sefidkon F, Goltaph E (2012). Effects of different nutrition systems (organic and chemical) on quantitative and qualitative characteristics of Fennel (Foeniculum vulgare, Mill.) under water deficit stress. Iran J Med Aromat Plants 2012; 28(2): 309-23.

Lawrence BM (1984). Progress in essential oils. Perf Flav; 9 (1): 59-60.

Mahmoud AM, El- Attar AB, Shehata SA (2016). Boosting fennel plant yield and components using combination of manure, compost and biofertilizers. Arabian Journal of Medicinal \& Aromatic Plants, Vol. 2 (1), p.: 28-36.

Mahmoud SAE (2009). Comparative study on black cumin (Nigella sativa, L.) plants grown under different spacing and fertilization treatments. M.Sc. Thesis, Faculty of Agriculture, Assiut University, Egypt.

Moradi R, Moghaddam PR, Mahallati NM, Nezhadali A (2011). Effects of organic and biological fertilizers on fruit yield and essential oil of sweet fennel (Foeniculum vulgare var. Dulce). Span J. of Agric. Res., 9 (2): 546-553.

Mounika Y, Sivaram GT, Reddy PSS, Ramaiah M (2018). Influence of biofertilizers and micronutrients on growth, seed yield and quality of coriander (Coriandrum sativum, L.) cv. Sadhana. Int.J.Curr.Microbiol.App.Sci (2018) 7(1): 20992107.

MSTAT-C (1986). A microcomputer program for the design management and analysis of Agronomic Research Experiments (version 4.0), Michigan State Univ., U.S.A.

Muthaura C, Musyimi DM, Joseph A, Okello S (2010). Effective microorganisms and their influence on growth and yield of pigweed (Amaranthus dubians). Arpn J. of Agric. and Biol. Sci., 5 (1): 1722.

Page AL, Miller RH, Keeney DR (1982) Methods of soil analysis; 2. Chemical and microbiological properties, 2. Aufl. 1184 S., American Soc. of Agronomy 
Patil NM (2010). Biofertilizer effect on growth, protein and carbohydrate content in Stevia rebaudiana var. Bertoni. Recent Research in Sci. and Techil., 2 (10) :42-44.

Rekaby AM (2013). Improving the productively of coriander plants by the use of some unconventional treatments. Ph.D. Thesis, Fac. of Agric. Minia Univ.

Sharaf MS, Khattab MF (2004). Effect of fertilization with inorganic, organic and biofertilizer on growth, yield and volatile oil concentration of fennel (Foeniculum vulgare, Mill) plants. J. Agric. Sci. Mansoura Univ., 29 (9): 5245-5264.

Suresh KD, Sneh G, Krishn KK, Mool CM (2004). Microbial biomass carbon and microbial activities of soils receiving chemical fertilizers and organic amendments. Arch. Agron. Soil Sci., 50: 641-647.

Tanious CTS (2008). Effect of some organic fertilization treatments on fennel plants. M. Sc. Thesis, Fac. Agric., Minia Univ., Egypt.

Younesian A, Taheri S, Moghaddam PR (2013). The effect of organic and biological fertilizers on essential oil content of Foeniculum vulgare, Mill. (Sweet Fennel). International Journal of Agriculture and Crop Sciences, IJACS, 5-18, 2141-2146.

Zand A, Darzi MT, Hadi MR (2013). Effects of phosphate solubilizing microorganisms and plant density of seed yield and essential oil content of anise (Pimpinella anisum). Middle - East Journal of Scientific Research, 14 (7): 940-946.

$$
\begin{aligned}
& \text { الملخص العربي } \\
& \text { تأثير التسميد العضوى والحيوى على نبات الثمر البلدى تحت نظام الرى بالتنقيط فى الواحات البحرية. } \\
& \text { II- إنتاجية الزيت وبعض الصفات الكيميائية } \\
& \text { " محمود عبدالهادي حسن عبده، "طارق عبدالناصر حلمى، "محمود صبحى سلام، " "أمير فتح الباب أحمد عبدالرحيم، "أحمد على حسن. } \\
& \text { "قسم البساتين - كلية الزراعة - جامعة المنيا- مصر } \\
& \text { " مركز التجارب والبحوث الزراعية - جامعة المنيا - مصر }
\end{aligned}
$$

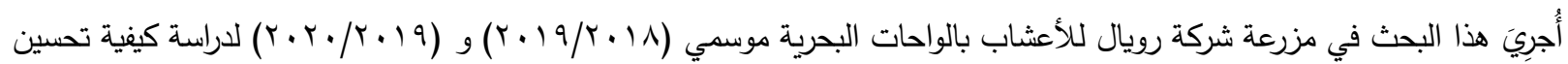

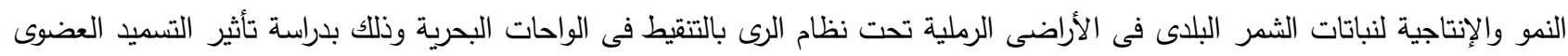

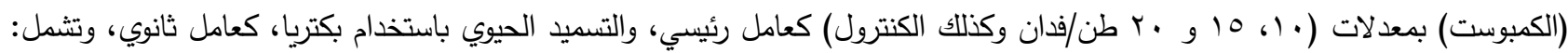

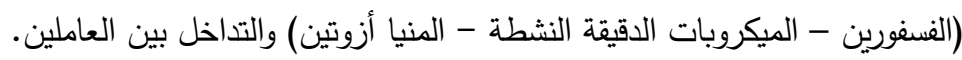

$$
\begin{aligned}
& \text { وبعد التجربة لموسمين زراعيين متتاليين، تم الحصول على تأثيرات إيجابية فى جميع معاملات الكمبوست والتسميد الحيوي علي صفات } \\
& \text { النسبة المئوية للزيت ومحصول الزيت للنبات والفدان وصبغات البناء الضوئى والنسبة المئوية لكل من النيتروجين والفوسفور والبوتاسيوم، خلال } \\
& \text { موسمي النمو. }
\end{aligned}
$$

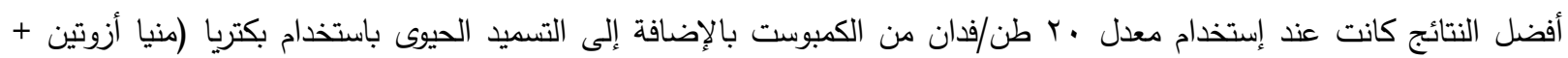

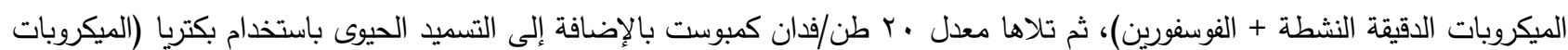

$$
\begin{aligned}
& \text { الدقيقة النشطة + الفوسفورين). }
\end{aligned}
$$

